\title{
Effectiveness of Different Doses of Diatomaceous Earth on Mexican Bean Weevil (Zabrotes subfasciatus Boheman) in Culiacan, Sinaloa, Mexico
}

\section{Jacobo Enrique Cruz Ortega ${ }^{1}$, Leopoldo Partida Ruvalcaba ${ }^{2 *}$, Teresa de Jesús Velásquez Alcaraz ${ }^{1}$, Juan Eulogio Guerra Liera1, Tomás Díaz Valdés ${ }^{1}$, Luz del Carmen Oliva Ortiz ${ }^{1}$}

${ }^{1}$ Facultad de Agronomía, Universidad Autónoma de Sinaloa, Culiacán de Rosales, México

${ }^{2}$ Universidad Tecnológica de Culiacán, Culiacán de Rosales, México

Email: *parpolo@yahoo.com.mx

How to cite this paper: Cruz Ortega, J.E., Partida Ruvalcaba, L., Alcaraz, T.J.V., Liera, J.E.G., Valdés, T.D. and Ortiz, L.C.O. (2016) Effectiveness of Different Doses of Diatomaceous Earth on Mexican Bean Weevil (Zabrotes subfasciatus Boheman) in Culiacan, Sinaloa, Mexico. Open Access Library Journal, 3: e3228.

http://dx.doi.org/10.4236/oalib.1103228

Received: November 14, 2016

Accepted: December 3, 2016

Published: December 6, 2016

Copyright $\odot 2016$ by authors and Open Access Library Inc.

This work is licensed under the Creative Commons Attribution International

License (CC BY 4.0).

http://creativecommons.org/licenses/by/4.0/

\section{Abstract}

The objective of this research was to determine the effectiveness of different doses of diatomaceous earth (TD) against bean Mexican weevil Zabrotes subfasciatus Boheman. An experiment was carried out in two phases: in first one tested diatomaceous earth at doses of 1.0, 2.0, 3.0, 4.0 and $5.0 \mathrm{~g} \cdot \mathrm{kg}^{-1}$ of seed, with samples at $15,30,45$ and 60 days after application (dda), while in the second the doses were $0.2,0.4,0.6,0.8$ and $1.0 \mathrm{~g} \cdot \mathrm{kg}^{-1}$ and samples at $10,20,30$ and $40 \mathrm{dda}$. The parameters evaluated were weevil mortality and seed germination. The results indicated that the average mortality rate was $100 \%$ where deltamethrin and diatomaceous earth were applied at 4.0 and $5.0 \mathrm{~g} \cdot \mathrm{kg}^{-1}$ of seed, with the $3.0 \mathrm{~g} \cdot \mathrm{kg}^{-1}$ dose was $97.6 \%$, with $2.0 \mathrm{G} \cdot \mathrm{kg}^{-1}$ was $97.0 \%$, with $1.0 \mathrm{~g} \cdot \mathrm{kg}^{-1}$ reaching $94.4 \%$, whereas in absolute control mortality was $1.2 \%$ $2.5 \%$. A similar response was observed with lower doses of diatomaceous earth $(0.6$, 0.8 and $1.0 \mathrm{~g} \cdot \mathrm{kg}^{-1}$ ) and the chemical Deltamethrin, which caused $100 \%$ mortality, with a dose of $0.4 \mathrm{~g} \cdot \mathrm{kg}^{-1}$ was $90 \%$ - $98 \%$, with $2.0 \mathrm{~g} \cdot \mathrm{kg}^{-1}$ from $62 \%$ to $83 \%$, whereas in the absolute control there was no mortality. The treatments did not inhibit seed germination in the two experimental phases, whose percentage ranged from $94 \%$ to $96 \%$.

\section{Subject Areas}

Agricultural Science

\section{Keywords}

Sampling, Mortality, Deltamethrin, Adult Weevil, Control 


\section{Introduction}

El frijol es una de las leguminosas más sembradas y consumidas en México; durante el ciclo agrícola 2014-2015 se sembraron 220,263 ha ${ }^{-1}$, correspondiéndole al estado de Sinaloa $58,550 \mathrm{ha}^{-1}$, para ubicarse en el primer lugar en cuanto a siembra y cosecha de este grano [1]. El frijol es uno de los alimentos esenciales para la población mundial, lo que hace necesario la conservación y protección de este grano contra los diversos factores que lo afectan; dentro de estos destaca por su importancia el gorgojo mexicano (Zabrotes subfasciatus Boheman, Coleoptera: Bruchidae) del frijol; la larva se alimenta del grano causando severo daño y provocando baja en el poder germinativo de la semilla, al dañar de manera considerable los cotiledones, también debido al ataque en la testa del grano se observan las oviposturas de la hembra y perforaciones que son las cámaras de alimentación de estos insectos [2] [3].

Las plagas de almacén constituyen uno de los problemas más importantes en el almacenamiento de granos y semillas, si no son controladas de manera oportuna, y ocasionan daños directos que repercuten en la conservación del grano; asimismo, daños indirectos al ser invadidos por diversos microorganismos como hongos y bacterias que los contaminan, que al ser consumidos pueden ocasionar problemas en la salud humana. Son pocos los productos que pueden utilizarse de manera confiable para el control de las plagas de los granos almacenados, principalmente insecticidas y fumigantes que son poco persistentes en México y en el mundo, lo que hace necesario buscar más alternativas para disminuir los daños que causan las plagas de los granos almacenados, que no dañen al medio ambiente y la salud humana. A nivel mundial se han probado diferentes alternativas para el control de las plagas de almacén, dentro de las cuales se mencionan tratamientos a base de calor y frío, el uso de extractos vegetales y sustancias minerales, feromonas como sustancias de atracción, control biológico y el uso de sustancias químicas que preferentemente tengan bajo poder residual y no provoquen efectos en los granos, semillas y consumidores [4] [5] [6] [7].

Martínez et al. [8] mencionan que en condiciones de almacenamiento los insectos más importantes que afectan al frijol son coleópteros de la familia Bruchidae, reportándose en México al gorgojo común del frijol (Acanthoscelides obtectus Say.) y al gorgojo mexicano del frijol (Zabrotes subfasciatus Boheman), los cuales ocasionan pérdidas de hasta $20 \%$ de la cosecha. Contra la última especie, Borboa et al. [9] probaron la actividad tóxica de diez polvos minerales y encontraron que la cal, caolín, cantera y mármol, provocaron mayor mortalidad, disminuyeron oviposición, daño y emergencia de adultos de esta plaga.

El empleo de sustancias naturales y minerales en el control de plagas de granos almacenados es una alternativa viable para el control de plagas como Sitophilus zeamais, Zabrotes subfasciatus y el gorgojo del garbanzo Callosobruchus maculatus y plantas como el chicalote Argemone sp. es un insecticida natural para el control de gorgojo mexicano del frijol ya que ocasiona mortalidades superiores al 97\% [10]. Otros trabajos que han mostrado buenos resultados del uso de plantas para el control de plagas de granos almacenados, son los realizados por Hincapié et al. [11], en donde 
señalan que extractos de semilla de Annona muricata controlan de manera satisfactoria a Sitophilus zeamais, ya que produce mortalidad de adultos, y la emergencia se inhibió en $100 \%$ a partir de las concentraciones de $2,500 \mathrm{ppm}$ para los extractos obtenidos con acetato de etilo y hexano y de 5,000 ppm para los obtenidos con alcohol etílico; los resultados indican que los extractos son más efectivos por ingestión que por contacto.

Valdés et al. [12], al evaluar siete especies distintas de plantas contra Zabrotes subfasciatus Boheman encontraron que los polvos de neem (Azadirachta indica Jauss.), caisimón de anís (Piper auritum Kunth.) y apazote (Chenopodium ambrosioides L.) al ser mezclados con la semilla de frijol inhibieron totalmente la oviposición de la plaga.

Nivea et al. [13] demostraron que los aceites esenciales de Eucalyptus Citridiora, E. staigeriana, Cymbopogon winterianus y Foeniculum vulgare controlan satisfactoriamente a Callosobruchus maculatus, y se determinó que a mayor concentración de aceite es menor la oviposición y, por tanto, menor será la emergencia de adultos de este insecto.

Ascencio [14] probó la actividad insecticida y repelente de dos extractos de Annona diversifolia (anona) contra Zabrotes subfasciatus Boheman, y encontró que tanto el extracto etanólico como el de n-hexáno: éter etílico (1:1) tuvieron acción insecticida y repelente contra esta plaga de almacén; sin embargo, fue el extracto etanólico a dosis de $300 \mathrm{ppm}$ con el que mejores resultados se obtuvieron, tanto para mortalidad de insecto como para efecto disuasivo, y por tanto menor oviposturas en el grano de frijol.

Tonielo et al. [15] describieron métodos alternativos para formular extractos botánicos de Azadirachta indica (neem), para mejorar su estabilidad biológica en el control de plagas, y mencionan que es importante formular los ingredientes que causan daño en los insectos, con niveles reproductivos de compuestos activos y por el uso de herramientas analíticas en programas de control de calidad; con nanopartículas cargadas con productos de neem y enriquecidos con extractos botánicos, se prepararon diferentes cantidades de azadiractina y fueron probados contra Zabrotes subfasciatus, los resultados indicaron mayor estabilidad en comparación con los productos comerciales. El uso de polvos minerales constituye una alternativa viable e importante en el control de los insectos de almacén, ya que no contamina el grano ni al ambiente, además al ser aplicado puede controlar insectos hasta por más de seis meses sin necesidad de volver a realizar otra aplicación.

La tierra de diatomea (TD) está constituida por dióxido de silicio $\left(\mathrm{SiO}_{2}\right)$ de restos fosilizados de diatomeas de agua dulce y salada [16]. $\mathrm{El} \mathrm{SiO}_{2}$ constituye cerca del 70\% 90\% del total de los compuestos presentes en las tierras de diatomeas, el resto son pequeñas cantidades de minerales, tales como, cristales de silicio, calcio, fósforo, azufre, niquel, zinc, manganeso, aluminio, óxido de hierro, magnesio, sodio, cal. Dependiendo del contenido de minerales es su color, el cual adquiere una tonalidad que va de blancogris a amarillo-rojo [7] [16] [17] [18]. Existen dos tipos de formulaciones de TD, las de agua dulce y las marinas. Las formulaciones marinas contienen una ligera concentración más alta de cristales de silicio ( $2 \%$ a $7 \%$ ) que la formulación de agua dulce $(1.0 \%$ o menos) [18]. Las diferentes formulaciones de TD pueden presentar una variación en la 
toxicidad. Esta variación se debe en gran parte a las características físicas del producto, las cuales dependen de la fuente donde se obtuvieron [4].

Sousa et al. [19], evaluaron el efecto de diferentes dosis de TD y temperaturas contra gorgojo del maíz y los resultados encontrados, mostraron que la mortalidad de $S$. zeamais aumentó con la dosis y temperatura más alta durante el período de exposición de seis y 15 días, estos mismos resultados fueron observados en la emergencia de la progenie, por lo que se asume que el aumento de la temperatura y el periodo de exposición favorece la eficacia de la TD incluso a dosis bajas en el control de esta plaga de almacén.

Quarles [20], señala que el frijol almacenado en sacos de $50 \mathrm{~kg}$ se puede proteger con cantidades tan pequeñas como $300 \mathrm{ppm}$ de tierra de diatomeas. Al aplicarse este mineral como un protector de superficie sin alimento (granos) a dosis de $0.5 \mathrm{mg} / \mathrm{cm}^{2}$, en ensayos de laboratorio se obtiene el 100\% de mortalidad en T. confusum [4].

Mazzuferi et al. [21], señalan que para determinar la efectividad de tres dosis de tierra diatomea $\left(0.5,1.0\right.$ y $2.0 \mathrm{~kg}^{-1}$ tonelada de semilla de maíz) sola y combinada con deltametrina sinergizada con butóxido de piperonilo, la tierra de diatomea a dosis de $2.0 \mathrm{~kg}^{-1}$ tonelada de semilla aplicada de manera individual causó mortalidades superiores al 97\% de gorgojo del maíz hasta los 120 días después de su aplicación y este mismo comportamiento se observó reflejado cuando fue combinada con el insecticida deltametrina.

Se evaluaron bajo condiciones de laboratorio polvo de semilla de neem, hoja de Plectranthus glandulosus, polvo de tierra de diatomeas (SilicoSec), aplicado en cuatro intervalos de exposición (1, 3, 7 y 14 días) para el control del gorgojo del maíz Sitophilus zeamais Motschulsky: los resultados mostraron que el tratamiento con SilicoSec fue el más eficaz, seguido de polvo de semilla de neem y polvo de $P$. Glandulosus $\left(2.0 \mathrm{~g} \cdot \mathrm{kg}^{-1}\right)$, con una mortalidad de adultos de gorgojo del maíz de 81.1 y $100 \%$ a los 3 y 14 días de exposición, respectivamente; seguido del polvo de neem $\left(40 \mathrm{~g} \cdot \mathrm{kg}^{-1}\right)$ con 86.8 y $59.5 \%$ de mortalidad, respectivamente. Los tratamientos redujeron la emergencia de la progenie, porcentaje de daño al grano, porcentaje de pérdida de peso y el porcentaje de pérdida de germinación [22].

De acuerdo con Yatie et al. [23], en un experimento realizado para probar el efecto de partículas minerales contra Zabrotes subfasciatus Boheman, observaron que el Kaolín a dosis de $8.0 \mathrm{~g} \cdot \mathrm{kg}^{-1}$ provocó mortalidades del $100 \%$ a partir de los cuatro y hasta los ocho días después de la aplicación, y que con el tratamiento a base de tierra de diatomea aplicado a dosis de $1.0 \mathrm{~g} \cdot \mathrm{kg}^{-1}$ el $100 \%$ de mortalidad se alcanzó a partir del tercero y hasta los ocho días posteriores; el tratamiento a base de Kaolín mostró una mortalidad de $40 \%$ al tercer día de evaluación y $92 \%$ en la evaluación registrada a los ocho días.

Se evaluó la efectividad de tres bioinsecticidas (Spinosad, Beauveria bassiana y Metarhizium anisopliae), tierra de diatomea y dióxido de carbono como una alternativa de control contra Rizopertha dominica, y los resultados indican que el Spinosad a dosis de $0.5 \mathrm{mg} \cdot \mathrm{kg}^{-1}$ y la tierra de diatomea a dosis de 0.8 y $1.6 \mathrm{~g} \cdot \mathrm{kg}^{-1}$ de semilla, mostraron una efectividad de $100 \%$ de control sobre esta plaga (Fawzy et al. [24]. 
La eficacia de tierra de diatomea (TD) fue evaluada contra tres plagas de maíz almacenado: Sitophilus zeamais, Tribolium castaneum y Palorus subdepressus a cuatro diferentes dosis $\left(1.5,3.0,4.5\right.$ y $\left.6.0 \mathrm{~g} \cdot \mathrm{kg}^{-1}\right)$ comparadas con cuatro dosis de Actellic de Super $^{\mathrm{ma}}$, un insecticida químico usado como control $\left(0.25,0.5,0.75\right.$ y $\left.1.0 \mathrm{~g} \cdot \mathrm{kg}^{-1}\right)$; los resultados encontrados demostraron que la TD fue tan eficaz como Actellic de Super ${ }^{\mathrm{ra}} \mathrm{y}$ que por lo tanto es una buena alternativa para el control de estas tres plagas de insectos de maíz y puede incorporarse fácilmente en los programas de manejo integrado de plagas de productos almacenados (Doumbia et al. [25].

En un trabajo donde se evaluó la eficacia de la tierra diatomea de depósitos de Grecia y Rumania sobre cuatro especies de insectos que afectan los granos almacenados: Tribolium castaneum (Herbst) (Coleoptera:Tenebrionidae), Oryzaephilus surinamensis (L.) (Coleoptera:Silvanidae), Sitophilus oryzae (L.) (Coleoptera: Curculionidae) y Rhyzopertha dominica (F.) (Coleoptera: Bostrychidae) a las dosis de 100, 300, 500 y 900 ppm; dos formulaciones naturales griegas (Elassona 1 y 2), dos rumanas (PatRom y AdRom) y dos comerciales (SilicoSec y PyriSec), se evaluó la mortalidad de los adultos a los 7, 14 y 21 días después de la aplicación, los resultados obtenidos muestran que PyriSec mostró $100 \%$ de mortalidad de O. surinamensis a dosis de 300 ppm a los 7 y 14 días de exposición al mineral (Athanassiou et al. [26].

Se evaluó la eficacia de una formulación de tierra de diatomeas (DiaFil $\left.{ }^{\oplus} 610\right)$ a tres diferentes dosis $\left(0,2.5\right.$ y $\left.5.0 \mathrm{~g} \cdot \mathrm{m}^{-2}\right)$ para el control de adultos de $T$. castaneum a cinco temperaturas constantes $\left(28^{\circ} \mathrm{C}, 36^{\circ} \mathrm{C}, 42^{\circ} \mathrm{C}, 44^{\circ} \mathrm{C} \mathrm{y} 46^{\circ} \mathrm{C}\right)$; diez adultos de este insecto se colocaron en recipientes tratados y no tratados con diatomea y con un periodo de exposición de 4, 8, 12, y 24 h en cada uno de las cinco temperaturas. La eficacia de la TD contra los adultos de $T$. castaneum fue mejor a temperaturas y periodo de exposición más altos. En general, más adultos murieron a la dosis de $5.0 \mathrm{~g} / \mathrm{m}^{2}$ con un $100 \%$ de mortalidad; en comparación con la de $2.5 \mathrm{~g} \cdot \mathrm{m}^{-2}$. Que observó una mortalidad de $73 \%, 1 \%, 77 \%$ a las 24 horas de exposición. También las temperaturas de $44^{\circ} \mathrm{C}$ y $46^{\circ} \mathrm{C}$, mostraron $100 \%$ de mortalidad de adultos después de $24 \mathrm{~h}$ de exposición; por lo tanto sugieren que el uso combinado de $\mathrm{DE}$ y temperaturas por debajo de $50^{\circ} \mathrm{C}$ se puede utilizar como un enfoque integrado para el control de plagas de insectos almacenados en el grano cosechado recientemente [27]. El objetivo del presente estudio fue determinar la efectividad de diferentes dosis de tierra de diatomea contra gorgojo mexicano del frijol Zabrotes subfasciatus Boheman, en Culiacán, Sinaloa, México.

\section{Materiales y Métodos}

El experimento se llevó a cabo en el Laboratorio de Entomología de la Facultad de Agronomía de la Universidad Autónoma de Sinaloa, ubicada en la carretera CulicacánEldorado kilómetro 17.5 en Culiacán, Sinaloa. Para realizar esta investigación se procedió a purificar la colonia del gorgojo pinto del fríjol (Zabrotes subfasciatus Boheman) en frascos de vidrio con capacidad de cinco kilogramos, los cuales se mantuvieron bajo una temperatura que fluctuó entre $30^{\circ} \mathrm{C}-35^{\circ} \mathrm{C}$, con el propósito de contar con una colonia homogénea para la prueba. 
Para establecer los ensayos se usaron vasos de poliestireno con capacidad de $500 \mathrm{~g}$, así como dos kilogramos de frijol por tratamiento; la aplicación de la tierra de diatomea se hizo homogeneizando ésta sobre el grano, posteriormente se depositaron 20 adultos de gorgojo pinto del frijol en cada repetición y se taparon con tela de organza. La experimentación se efectuó en dos fases: a) la primera se hizo en un diseño completamente al azar con siete tratamientos y cuatro repeticiones; los tratamientos fueron tierra de diatomea en dosis de $1.0,2.0,3.0,4.0$ y $5.0 \mathrm{~g} \cdot \mathrm{kg}^{-1}$ de semilla, un testigo químico (Deltametrina) en dosis de $1.0 \mathrm{~mL} \cdot \mathrm{kg}^{-1}$ de semilla, más un testigo absoluto (sin aplicación de sustancias); b) la segunda fase consistió en otro diseño experimental completamente al azar con la misma cantidad de tratamientos y repeticiones, pero con las dosis de $0.2,0.4 .0 .6,0.8$ y $1.0 \mathrm{~g} \cdot \mathrm{kg}^{-1}$ de tierra diatomea, un testigo químico (Deltametrina) en dosis de $0.1 \mathrm{~mL} \cdot \mathrm{kg}^{-1}$ de semilla, más el testigo absoluto.

En ambas fases de la experimentación las variables de respuesta fueron el porcentaje de adultos muertos y la germinación de semillas de frijol. En la primera fase la mortalidad se determinó con el número de insectos vivos y muertos en cada unidad experimental, a los 15, 30, 45 y 40 días después de la aplicación (dda), mientras que en la segunda fase se hizo a los 10, 2030 y 40 dda. Con los promedios de mortalidad en cada unidad experimental se obtuvo el porcentaje de efectividad aplicando la fórmula de Abbott [28]:

$$
\text { Mortalidad corregida }=\frac{\text { Mortalidad del tratamiento }- \text { Mortalidad del testigo } \times 100}{100-\text { Mortalidad del testigo }}
$$

La germinación se evaluó con 100 granos de frijol sembrados en charolas de poliestireno rellenas con peat moss, y se determinó a los 10, 20 y 30 dda de las dosis de tierra de diatomea y Deltametrina, al contar las plántulas emergidas en cada una de las unidades experimentales y comparación de promedios en relación al testigo, mientras que los porcentajes también se determinaron con la fórmula de Abbott [29]. Todos los datos se sometieron a un análisis de varianza y comparación múltiple de medias con la prueba de Tukey ( $\alpha=0.05$ ), con el paquete estadístico SAS 9.1 [28].

\section{Resultados y Discusión}

Los resultados muestran que todas las dosis de tierra de diatomea (TD) ejercieron un excelente control, de tal forma que en las evaluaciones registradas a los 15 días después de la aplicación se presentaron mortalidades de $100 \%$ con las dosis de 4.0 y $5.0 \mathrm{~g} \cdot \mathrm{kg}^{-1}$, similares a las observadas con el testigo químico (Deltametrina); las dosis de 2.0 y 3.0 $\mathrm{g} \cdot \mathrm{kg}^{-1}$ de TD ocasionaron un porcentaje de mortalidad de adultos de Zabrotes subfasciatus de 95 y 96\%, respectivamente; la dosis de $1.0 \mathrm{~g}$ de TD provocó mortalidad de $93 \%$, sin que se dieran diferencias estadísticas entre los promedios logrados con las dosis de TD y Deltametrina (Table 1), aunque dichos promedios fueron significativamente diferentes con respecto al promedio observado en el testigo absoluto.

Este mismo comportamiento se observó en la evaluaciones registradas a los 30, $45 \mathrm{y}$ 60 dda (Table 1), donde se aprecia que todas las dosis de TD utilizadas para el control de la plaga provocaron mortalidades superiores al $90 \%$, esto indica que aunque el 
Table 1. Porcentaje de mortalidad de adultos de gorgojo del frijol (Zabrotes subfasciatus Boh.) tratados con tierra de diatomea (TD). Ciclo 2014-2015.

\begin{tabular}{ccccc}
\hline Tratamientos/dosis & $\begin{array}{c}\text { Mortalidad (\%) } \\
15 \mathrm{dda}\end{array}$ & $\begin{array}{c}\text { Mortalidad (\%) } \\
30 \mathrm{dda}\end{array}$ & $\begin{array}{c}\text { Mortalidad (\%) } \\
45 \mathrm{dda}\end{array}$ & $\begin{array}{c}\text { Mortalidad (\%) } \\
60 \mathrm{dda}\end{array}$ \\
\hline Testigo absoluto & $1.2 \mathrm{~b}^{*}$ & $1.2 \mathrm{~b}$ & $1.2 \mathrm{~b}$ & $2.5 \mathrm{~b}$ \\
Deltametrina, $1.0 \mathrm{~mL} \cdot \mathrm{kg}^{-1}$ & $100 \mathrm{a}$ & $100 \mathrm{a}$ & $100 \mathrm{a}$ & $100 \mathrm{a}$ \\
TD, $1.0 \mathrm{~g} \cdot \mathrm{kg}^{-1}$ & $93.0 \mathrm{a}$ & $93.0 \mathrm{a}$ & $93.0 \mathrm{a}$ & $98.7 \mathrm{a}$ \\
TD, $2.0 \mathrm{~g} \cdot \mathrm{kg}^{-1}$ & $95.0 \mathrm{a}$ & $97.5 \mathrm{a}$ & $97.0 \mathrm{a}$ & $98.7 \mathrm{a}$ \\
TD, $3.0 \mathrm{~g} \cdot \mathrm{kg}^{-1}$ & $96.0 \mathrm{a}$ & $97.5 \mathrm{a}$ & $97.0 \mathrm{a}$ & $100 \mathrm{a}$ \\
TD, $4.0 \mathrm{~g} \cdot \mathrm{kg}^{-1}$ & $100 \mathrm{a}$ & $100 \mathrm{a}$ & $100 \mathrm{a}$ & $100 \mathrm{a}$ \\
TD, $5.0 \mathrm{~g} \cdot \mathrm{kg}^{-1}$ & $100 \mathrm{a}$ & $100 \mathrm{a}$ & $100 \mathrm{a}$ & $100 \mathrm{a}$ \\
\hline
\end{tabular}

*Promedios con diferente letra en la columna son estadísticamente diferentes, según Tukey $(\alpha \leq 0.05)$; dda = días después de la aplicación.

periodo de exposición fue de dos meses, los porcentajes de mortalidad se mantuvieron en $100 \%$ con las dosis de $3.0,4.0$ y $5.0 \mathrm{~g} \cdot \mathrm{kg}^{-1}$ de TD y Deltametrina en dosis de 1.0 $\mathrm{mL} \cdot \mathrm{kg}^{-1}$ de semilla; las dosis más bajas $\left(1.0\right.$ y $\left.2.0 \mathrm{~g} \cdot \mathrm{kg}^{-1}\right)$ también ejercieron excelente control de Zabrotes subfasciatus Boh., con diferencias estadísticas de mortalidad sólo con relación al que se obtuvo en el testigo absoluto.

Los resultados del primer experimento sirvieron para tomar la decisión de realizar un segundo experimento con dosis menores que fueron: 0.2, 0.4, 0.6, 0.8 y $1.0 \mathrm{~g} \cdot \mathrm{kg}^{-1} \mathrm{de} T \mathrm{~T}$, $0.1 \mathrm{~mL} \cdot \mathrm{kg}^{-1}$ de Deltametrina (químico) y un testigo absoluto (sin aplicación). Los resultados indicaron que a los $10 \mathrm{dda}$ las dosis de TD de $0.6,0.8$ y $1.0 \mathrm{~g} \cdot \mathrm{kg}^{-1}$ de semilla ocasionaron mortalidad de $100 \%$ (Table 2), similar a la ocasionada por el testigo químico (Deltametrina), sin diferencias significativas entre los promedios; sin embargo, estos porcentajes de mortalidad fueron significativamente diferentes a los ocasionados con las dosis de 0.2 y $0.4 \mathrm{~g} \cdot \mathrm{kg}^{-1}$ de semillas, y más aún con respecto al porcentaje de mortalidad (0) que se tuvo en el testigo absoluto. A los 20 dda se observó que donde se aplicaron las dosis de $0.6,0.8$ y $1.0 \mathrm{~g} \cdot \mathrm{kg}^{-1}$ de TD y $0.1 \mathrm{~mL} \cdot \mathrm{kg}^{-1}$ de Deltametrina (químico), se tuvieron porcentajes de mortalidad de $100 \%$ de adultos de gorgojo del frijol, pero no fueron significativamente diferentes al $95 \%$ que se logró con la dosis de $0.4 \mathrm{~g} \cdot \mathrm{kg}^{-1}$ de TD; no obstante, si fueron estadísticamente diferentes a la mortalidad ( $28 \%$ menos) que se logró con la dosis de $0.2 \mathrm{~g} \cdot \mathrm{kg}^{-1}$ de TD; asimismo, con respecto al $0 \%$ que se observó en el testigo absoluto.

A los $40 \mathrm{dda}$ los tratamientos en dosis de $0.6,0.8$ y $1.0 \mathrm{~g} \cdot \mathrm{kg}^{-1} \mathrm{de}$ TD y $0.1 \mathrm{~mL} \cdot \mathrm{kg}^{-1} \mathrm{de}$ Deltametrina, la mortalidad fue del $100 \%$ (Table 2), sin diferencia significativa con la que ocasionó la dosis de $0.4 \mathrm{~g} \cdot \mathrm{kg}^{-1}$ de TD; sin embargo, dichos porcentajes fueron significativamente diferentes a la mortalidad que se produjo con la dosis de $0.2 \mathrm{~g} \cdot \mathrm{kg}^{-1}$ de TD y con el testigo absoluto.

La germinación de semillas fue similar con todos los tratamientos aplicados, incluido el testigo absoluto, con una emergencia de plántulas que osciló entre 94 y 96\%, considerada como normal, y se asume que la tierra de diatomea no provocó ningún efecto sobre la germinación. 
Table 2. Porcentaje de mortalidad de adultos de gorgojo del frijol (Zabrotes subfasciatus Boh.) tratados con dosis menores de tierra de diatomea (TD). Ciclo 2014-2015.

\begin{tabular}{ccccc}
\hline Tratamientos/dosis & $\begin{array}{c}\text { Mortalidad (\%) } \\
10 \mathrm{dda}\end{array}$ & $\begin{array}{c}\text { Mortalidad (\%) } \\
20 \mathrm{dda}\end{array}$ & $\begin{array}{c}\text { Mortalidad (\%) } \\
30 \mathrm{dda}\end{array}$ & $\begin{array}{c}\text { Mortalidad (\%) } \\
40 \mathrm{dda}\end{array}$ \\
\hline Testigo absoluto & $0.0 \mathrm{~d}^{*}$ & $0.0 \mathrm{c}$ & $0.0 \mathrm{c}$ & $0.0 \mathrm{c}$ \\
Deltametrina, $0.1 \mathrm{~mL} \cdot \mathrm{kg}^{-1}$ & $100 \mathrm{a}$ & $100 \mathrm{a}$ & $100 \mathrm{a}$ & $100 \mathrm{a}$ \\
TD, $0.2 \mathrm{~g} \cdot \mathrm{kg}^{-1}$ & $62.0 \mathrm{c}$ & $72.0 \mathrm{~b}$ & $79.0 \mathrm{~b}$ & $83.0 \mathrm{~b}$ \\
TD, $0.4 \mathrm{~g} \cdot \mathrm{kg}^{-1}$ & $90.0 \mathrm{~b}$ & $95.0 \mathrm{a}$ & $97.0 \mathrm{a}$ & $98.0 \mathrm{a}$ \\
TD, $0.6 \mathrm{~g} \cdot \mathrm{kg}^{-1}$ & $100 \mathrm{a}$ & $100 \mathrm{a}$ & $100 \mathrm{a}$ & $100 \mathrm{a}$ \\
TD, $0.8 \mathrm{~g} \cdot \mathrm{kg}^{-1}$ & $100 \mathrm{a}$ & $100 \mathrm{a}$ & $100 \mathrm{a}$ & $100 \mathrm{a}$ \\
TD, $1.0 \mathrm{~g} \cdot \mathrm{kg}^{-1}$ & $100 \mathrm{a}$ & $100 \mathrm{a}$ & $100 \mathrm{a}$ & $100 \mathrm{a}$ \\
\hline
\end{tabular}

${ }^{*}$ Promedios con diferente letra en la columna son estadísticamente diferentes, según Tukey $(\alpha \leq 0.05)$; dda $=$ días después de la aplicación.

Estos resultados coinciden con lo expuesto por Yatie et al. [22], donde se señala que la tierra de diatomea constituye un mineral con potencial insecticida contra el gorgojo del frijol, aplicada en dosis de $1.0 \mathrm{~g} \cdot \mathrm{kg}^{-1}$ para tener $100 \%$ de mortalidad de los tres a los ocho días posteriores a la aplicación. También coinciden con los de Cook y Armitage [15], Arthur [4], Korunic [6], Fields y Korunic [17], toda vez que refieren que estos polvos inertes se han empleado con gran éxito en el control de gran número de insectos de granos almacenados, entre los que se encuentran, Oryzaephilus surinamensis, $R$. dominica, Tribolium castaneum, T. confusum, Cryptolestes ferruguineus, $S$. zeamais, $S$. granarius, S. orizae. Prostephanus truncatus y Acanthocelides obtectus y Zabrotes subfasciatus. Asimismo, con los de Mazzuferi et al. [20], pues indican que dosis de tierra diatomea $(0.5,1.0$ y $2.0 \mathrm{~kg}$ por tonelada de semilla de maíz) sola y combinada con Deltametrina sinergizada con butóxido de piperonilo, provocan mortalidad superior al 97\% de gorgojo del maíz, hasta los 120 días después de su aplicación, y este mismo comportamiento se observó cuando fue combinada con el insecticida Deltametrina.

Además, concuerdan con los de Lazzari y Ribeiro [30], porque señalan que la tierra de diatomea es una alternativa para control de Zabrotes subfasciatus Boheman, ya que después de cinco días de exposición y temperaturas de $27^{\circ} \mathrm{C}$ y $30^{\circ} \mathrm{C}$ tuvieron mortalidad de $100 \%$ con todas las dosis aplicadas $\left(0.5,0.75\right.$ y $1.0 \mathrm{~g} \cdot \mathrm{kg}^{-1}$ de semilla), concluyendo que las dosis adecuadas para el control de esta plaga de almacén son las de 0.75 y $1.0 \mathrm{~g} \cdot \mathrm{kg}^{-1}$ de semilla.

También tienen relación con lo expuesto por Rumbos et al. [31], quienes señalan que polvos minerales como la zeolita pueden controlar plagas de granos almacenados, como Sitophilus oryzae, Tribolium confusum y Oryzaephilus surinamensis, y que por lo tanto este material puede usarse exitosamente como protector de granos y semillas. Asimismo, con lo reportado por Subramanyam y Roesly [32] y Silva et al. [33], ya que mencionan que los polvos inertes causan efectos abrasivos sobre la cutícula de los insectos, lo que trae como consecuencia una pérdida de agua $y$, por consiguiente, la muerte. Además, estos polvos pueden ser usados en combinación con otros productos como los 
polvos vegetales para aumentar de esta manera la efectividad del control de plagas.

\section{Conclusiones}

Las dosis de 0.8 a $5.0 \mathrm{~g} \cdot \mathrm{kg}^{-1}$ de tierra de diatomea controlaron eficazmente al gorgojo mexicano del frijol, pero la dosis que se recomienda es la de $0.8 \mathrm{~g} \cdot \mathrm{kg}^{-1}$ de semilla, toda vez que con esta dosis se puede controlar con sustentabilidad y no se afecta la germinación de semillas, como con las otras dosis evaluadas.

\section{References}

[1] SIAP (2016) Servicio de Información Agroalimentaria y Pesquera. www.gob.mx/siap/

[2] Centro Internacional de Agricultura Tropical (1986) Insectos del frijol almacenado y su control por C.I.A.T. Folleto de divulgación 1344, Apartado aéreo 6713, Cali, Colombia, 4 p.

[3] Soto, P.O.J. (2014) Evaluación de la resistencia de variedades de frijol común (Phaseolus vulgaris L.) al ataque de gorgojo del frijol Zabrotes subfasciatus Boheman. Tesis para obtener el grado de Agroindustria Alimentaria. Escuela Agrícola Panamericana, Zamorano, Honduras, 30 p. https://bdigital.zamorano.edu/bitstream/11036/3377/1/AGI-2014-T041.pdf

[4] Arthur, F.H. (2000) Toxicity of Diatomaceous Earth to Red Flour Beetles and Confused Flour Beetles (Coleoptera: Tenebrionidae): Effects of Temperature and Relative Humidity. Journal Economic Entomology, 93, 526-532. https://doi.org/10.1603/0022-0493-93.2.526

[5] Arthur, F.H. (2001) Immediate and Delayed Mortality of Oryzaephilus surinamensis (L.) Exposed on Wheat Treated with Diatomaceous Earth: Effects of Temperature, Relative Humidity, and Exposure Interval. Journal of Stored Products Research, 37, 13-21. https://doi.org/10.1016/S0022-474X(99)00058-2

[6] Korunic, Z. (1997) Rapid Assessment of the Insecticidal Value of Diatomaceous Earths without Conducting Bioassays. Journal of Stored Products Research, 33, 219-229. https://doi.org/10.1016/S0022-474X(97)00004-0

[7] Korunic, Z. (1998) Diatomaceous Earths, a Group of Natural Insecticides. Journal of Stored Products Research, 34, 87-97. https://doi.org/10.1016/S0022-474X(97)00039-8

[8] Martínez, G.M.A., Osuna, C.E.S., Padilla, R.J.S., Acosta, G.J.A. and Loredo, O.C. (2008) Tecnología para la Producción de Frijol en el Norte Centro de México. SAGARPA, CONACYT, INIFAP. Libro Técnico Número 4, 216 p. http://biblioteca.inifap.gob.mx:8080/xmlui/bitstream/handle/123456789/1375/783.pdf?sequ ence $=1$

[9] Borboa, F.J., Dávila, S.E., Wong, C.F.J. and Fu, C.A. (2005) Utilización de polvos minerales en frijol para el control de Zabrotes subfasciatus (Boheman) (Coleóptera: Bruchidae). In: Martínez, Eds., Tecnología para la Producción de Frijol en el Norte Centro de México, Libro Técnico Número 4, 216 p.

http://biblioteca.inifap.gob.mx:8080/xmlui/bitstream/handle/123456789/1375/783.pdf?sequ ence $=1$

[10] Cuevas, S.M.I., García, M.J.C. and Romero, N.C.A. (2006) Productos naturales para el control de la principal plaga de maíz, frijol y garbanzo almacenados. Boletín de la Asociación Española de Entomología, 30, 83-92.

http://www.entomologica.es/index.php?d=publicaciones\&num $=37$

[11] Hincapié, L.C.A., Lopera, A.D. and Ceballos, G.M. (2008) Actividad insecticida de extractos de semilla de Annona muricata (Anonaceae) sobre Sitophilus zeamais (Coleoptera: Curculionidae). Revista Colombiana de Entomología, 34, 76-82. 
[12] Valdés, H.R., Pozo, V.E., Moya, A.A. and Cárdenas, M.M. (2010) Efecto de siete especies de plantas sobre Zabrotes subfasciatus Boh. Revista Centro Agrícola, 37, 83-88.

[13] Nivea, M.S., G. De Oliveira, J.V., Navarro, D.D., Dutra, K.A., Da Silva, W.A. and Wanderley, M.J.A. (2013) Contact and Fumigant Toxicity and Repellency of Eucalyptus citriodora Hook., Eucalyptus staigeriana F., Cymbopogon winterianus Jowitt and Foeniculum vulgare Mill. Essential Oils in the Management of Callosobruchus Maculatus (FABR.) (Coleoptera: Chrysomelidae, Bruchinae). Journal of Store Products Research, 54, 41-47. https://doi.org/10.1016/j.jspr.2013.02.002

[14] Ascencio, A.J.I. (2012) Evaluación de la actividad insecticida y repelente del extracto obtenido a partir de la semilla de Anona diversifolia (Anona) sobre el Zabrotes subfasciatus (Gorgojo común del frijol). Tesis de Licenciatura en Química y Farmacia, Universidad del Salvador, Buenos Aires, 87 p. http://ri.ues.edu.sv/2061/2/EVALUACION_DE_LA_ACTIVIDAD_INSECTICIDA_Y_REP ELENTE_DEL_EXTRACTO_OBTENIDO_A_PARTIR_DE_LA_SEMILLA_D.pdf

[15] Tonielo, J.C., Rossi, M.F., Soares, E.C., Rodrigues, J.S., Marques, M.J. and Leal, A.B.J. (2014) Effects of Different Formulations of Neem Oil-Based Products on Control Zabrotes subfasciatus (Boheman, 1833) (Coleoptera: Bruchidae) on Beans. Journal of Stored Products Research, 56, 49-53. https://doi.org/10.1016/j.jspr.2013.10.004

[16] Cook, D.A. and Armitage, D.M. (2000) Efficacy of a Diatomaceous Earth against Mite and Insect Populations in Small Bins of Wheat under Conditions of Low Temperature and High Humidity. Pest Management Science, 56, 591-596. https://doi.org/10.1002/1526-4998(200007)56:7<591::AID-PS180>3.0.CO;2-L

[17] Golob, P. (1997) Current Status and Future Perspectives for Inert Dusts for Control of Stored Product Insects. Journal of Stored Products Research, 33, 69-79. https://doi.org/10.1016/S0022-474X(96)00031-8

[18] Fields, P. and Korunic, Z. (2000) The Effect of Grain Moisture Content and Temperature on the Efficacy of Diatomaceous Earths from Different Geographical Locations against Stored-Product Beetles. Journal of Stored Products Research, 36, 1-13. https://doi.org/10.1016/S0022-474X(99)00021-1

[19] Sousa, A.H., Faraoni, L.R.A., Andrade, S.G., Freitas, S.R. and Pimentel, A.G.M. (2013) Bioactivity of Diatomaceous Earth to Sitophilus zeamais (Coleoptera: Curculionidae) in Different Application Conditions. Revista Brasileira de Engenharia Agrícola e Ambiental, 17, 982-986. https://doi.org/10.1590/S1415-43662013000900011

[20] Quarles, W. (1992) Diatomaceous Earth for Pest Control. The IPM Practitioner, 14, 1-11.

[21] Mazzuferi, V.E., Goncalvez, R.H., Tablada, M. and García, D. (2006) Efectividad y persistencia de la tierra de diatomeas en el control de Sitophilus zeamais (Coleoptera: Curculionidae) en semillas de maíz y su incidencia en la calidad. Boletín Sanidad Vegetal Plagas, 32, 363-371.

[22] Nukenine, E.K., Goudoungou, J.W., Adler, C. and Reichmuth, C. (2010) Efficacy of Diatomaceous Earth and Botanical Powders against the Maize Weevil, Sitophilus zeamais Motschulsky (Coleoptera: Curculionidae) on Maize. 10th International Working Conference on Stored Product Protection, Estoril, 27 June-2 July 2010, 881-897.

[23] Yatie, A.M., PissinatiI, A., FagottiI, D., Menezes, A.O.J. and Ursi, M.V. (2014) Control of the Mexican Bean Weevil Zabrotes subfasciatus with Kaolin. Ciência Rural, 40, 1497-1501.

[24] Fawzy, I.E., Nour El-Hoda, A.Z., Mohamed, Y.H. and Sayeda, A. (2014) Insecticidal Efficacy of Certain Bio-Insecticides, Diatomaceous Earth and Modified Atmospheres against Rhyzopertha dominica (F.) (Coleoptera: Bostrichidae) on Stored Wheat. Journal of Stored Products Research, 57, 30-35. 
[25] Doumbia, M., Douan, B.G., Kwadjo, K.E., Kra, D.K., Martel, V. and Doumbia, D. (2014) Effectiveness of Diatomaceous Earth for Control of Sitophilus zeamais (Coleoptera: Curculionidae), Tribolium castaneum and Palorus subdepressus (Coleoptera: Tenebrionidae). Journal of Stored Products Research, 57, 1-5. https://doi.org/10.1016/j.jspr.2013.11.008

[26] Athanassiou, G.C., Kavallieratos, N.G., Chiriloaie, A., Vassilakos, N.T., Fatu, V., Ciobanu, M. and Dudo, R. (2016) Insecticidal Efficacy of Natural Diatomaceous Earth Deposits from Greece and Romania against Four Stored Grain Beetles: The Effect of Temperature and Relative Humidity. Bulletin of Insectology, 69, 25-34.

[27] Frederick, L.J. and Subramanyam, B. (2016) Influence of Temperature and Application Rate on Efficacy of a Diatomaceous Earth Formulation against Tribolium castaneum Adults. Journal of Stored Products Research, 69, 86-90. https://doi.org/10.1016/j.jspr.2016.06.009

[28] Abbott, W.S. (1925) A Method of Computing the Effectiveness of an Insecticide. Journal Economic Entomology, 18, 265-267. https://doi.org/10.1093/jee/18.2.265a

[29] SAS Institute Inc. (1998) SAS/STAT ${ }^{\circledast} 9.1$ User’s Guide. SAS Institute Inc., Cary, 1028 p.

[30] Lazzari, F.N. and Ribeiro, C.C.S. (2006) Control of Zabrotes subfasciatus (Boheman) (Coleoptera, Chrysomelidae, Bruchinae) in Phaseolus vulgaris Linnaeus, Using Diatomaceous Earth under Different Temperatures. 9th International Working Conference on Stored Product Protection, Campinas, 15-18 October 2006, 804-810.

[31] Rumbos, C.I., Sakkab, M., Berillisc, P.C. and Athanassiou, G. (2016) Insecticidal Potential of Zeolite Formulations against Three Stored-Grain Insects, Particle Size Effect, Adherence to Kernels and Influence on Test Weight of Grain. Journal of Stored Products Research, 68, 93-101. https://doi.org/10.1016/j.jspr.2016.05.003

[32] Subramanyam, B., and Roesli, R. (2000) Inert Dust. In: Subramanyamy, B.D. and Hagstrum, W. Eds., Alternatives to Pesticides in Stored Product IMP, Kluwer Academic Publishers, Boston, 321-380. https://doi.org/10.1007/978-1-4615-4353-4_12

[33] Silva, A.G., González, G.P., Hepp, G.R. and Casals, B.P. (2004) Control de Sitophilus zeamais Motschulsky con polvos inertes. Agrociencia, 38, 529-536.

\section{Submit or recommend next manuscript to OALib Journal and we will provide best service for you:}

- Publication frequency: Monthly

- 9 subject areas of science, technology and medicine

- Fair and rigorous peer-review system

- Fast publication process

- Article promotion in various social networking sites (LinkedIn, Facebook, Twitter, etc.)

- Maximum dissemination of your research work

Submit Your Paper Online: Click Here to Submit

Or Contact service@oalib.com 